\title{
Dopaminergic Regulation of Reward System Connectivity Underpins Pain and Emotional Suffering in Migraine
}

\author{
Dajung J Kim (ID) \\ Hassan Jassar \\ Manyoel Lim \\ Thiago D Nascimento \\ Alexandre F DaSilva \\ Headache and Orofacial Pain Effort \\ (H.O.P.E.), Biologic and Material \\ Sciences \& Prosthodontics, University of \\ Michigan School of Dentistry, Ann \\ Arbor, MI, 48I09, USA
}

This article was published in the following Dove Press journal:
Journal of Pain Research

\begin{abstract}
Purpose: It has been suggested that reward system dysfunction may account for emotion and pain suffering in migraine. However, there is a lack of evidence whether the altered reward system connectivity is directly associated with clinical manifestations, including negative affect and ictal pain severity and, at the molecular level, the dopamine (DA) D2/D3 receptors (D2/3Rs) signaling implicated in encoding motivational and emotional cues.
\end{abstract}

Patients and Methods: We acquired resting-state functional MRI from interictal episodic migraine (EM) patients and age-matched healthy controls, as well as positron emission tomography (PET) with $\left[{ }^{11} \mathrm{C}\right]$ raclopride, a selective radiotracer for DA D2/3Rs, from a subset of these participants. The nucleus accumbens (NAc) was seeded to measure functional connectivity (FC) and DA D2/3Rs availability based on its essential involvement in pain-related aversive/reward functions. Associations of the brain measures with positive/ negative affect and ictal pain severity were also assessed.

Results: Compared with controls, the EM group showed weaker right NAc connectivity with areas implicated in pain and emotional regulation, such as the amygdala, rostral anterior cingulate cortex, hippocampus, and thalamus; but showed stronger left NAc connectivity with the dorsolateral prefrontal cortex and lingual gyrus. Moreover, among the altered NAc connectivities, only right NAc-amygdala connectivity was inversely correlated with DA D2/3Rs availability in migraine patients (diagnostic group-by-D2 /3Rs interaction $\mathrm{p}<0.007$ ). At a clinical level, such weaker NAc-amygdala connectivity was associated with lower interictal positive affect and greater ictal pain severity over the head and facial extension area (pain area and intensity number summation, PAINS).

Conclusion: Together, our findings suggest that altered reward system connectivity, specifically between the NAc and amygdala, might be affected by endogenous DA D2/3Rs signaling, and such process might be a neural mechanism that underlies emotional and pain suffering in episodic migraineurs.

Keywords: dopamine, nucleus accumbens, functional connectivity, migraine, reward system, craniofacial pain

\section{Introduction}

Migraine is a devastating neurological disorder characterized by recurrent episodes of headache attacks with gastrointestinal symptoms (eg, nausea, vomiting) and hypersensitivity to odors, touch, light, and sound. ${ }^{1}$ These symptoms are often accompanied by allodynia in $63 \%$ of patients during a migraine attack. ${ }^{2}$ In addition
Headache and Orofacial Pain Effort Lab O.P.E.), Biologic and Material Sciences \& Prosthodontics, University of Michigan School of Dentistry, I0II N University Avenue, Ann Arbor, MI, 48109, USA

Tel +l 7346153807

Fax +I 7347633453

Email adasilva@umich.edu
Journal of Pain Research 2021:14 63I-643 
to pain, emotional dysregulation such as depression and anxiety is another burden that translates migraine into a more hard-to-treat disorder. ${ }^{3}$

Long-term repeated migraine attacks are chronic stressors that reorganize the brain regions involved in pain and emotion. ${ }^{4}$ Alongside these changes, studies point to abnormal reward processing in episodic migraine (EM) patients even during the interictal period, such as altered neural response to secondary (eg, monetary) reward/loss consumption. ${ }^{5}$ Seemingly unrelated, it is evident that reward strongly interacts with pain through their overlapping neuroanatomical structures such as the amygdala and nucleus accumbens (NAc). ${ }^{6,7}$ Chronic pain impairs the mesolimbic reward system, which leads to reduced motivated behaviors, negative affect, and further debilitating pain-related suffering. ${ }^{6-8}$ However, neuroimaging evidence regarding an association between the reward system and clinical symptoms in migraine is very limited.

Specifically, the NAc receives dopaminergic input from the ventral tegmental area, which constitutes the basis of the mesolimbic reward circuitry. ${ }^{9}$ Also, it plays a significant role in various pain experiences, including pain aversiveness and stress, ${ }^{10,11}$ reward from pain relief, ${ }^{12,13}$ and chronification of pain. ${ }^{14,15}$ The involvement of the NAc in emotional and motivational processing in pain context can be possible through its functional connection with brain regions responsible for affective and executive functions such as the prefrontal cortex and anterior cingulate cortex (ACC). ${ }^{16}$ Thus, we hypothesized that the reward system constructed by NAc connectivity would be compromised and, further, might be relevant for affect and pain suffering in migraine patients.

Of note, researchers suggested that dopamine (DA), a key neurotransmitter involved in the reward and aversive learning, modulates the NAc pathway through D2 type receptor signalings. ${ }^{10}$ Recent multimodal neuroimaging studies have reported that experimental or endogenous DA was associated with brain signal variability, functional connectivity (FC), and between-network coupling. ${ }^{17-21}$ Thus, we hypothesized that endogenous DA receptor signaling would be associated with NAc connectivity in migraine. However, no studies have investigated these relationships in interictal EM patients to date.

We, therefore, investigated whether migraine exhibits an alteration in NAc connectivity during the interictal (headache-free) phase, if so, whether altered NAc connectivity is associated with endogenous DA receptor availability. To do so, we acquired resting-state functional
Magnetic Resonance Imaging (rs-fMRI) and Positron Emission Tomography (PET) with radiotracer $\left[{ }^{11} \mathrm{C}\right]$ raclopride, a DA D2/D3 receptors (D2/3Rs) antagonist ligand, from interictal EM patients and healthy controls $(\mathrm{HC})$. We seeded the NAc for measuring both FC and DA D2/3Rs availability. We further examined whether these molecular or functional brain properties would be associated with positive or negative affect and ictal clinical pain severity.

\section{Patients and Methods Study Participants}

We recruited participants, including patients and controls, through local advertising in the university's surrounding area. The age range was 18-45 years. The diagnosis of EM was determined by the International Classification of Headache Disorders (ICHD-3-beta). ${ }^{22}$ Specific exclusion criteria were applied to both patients and controls as follows: the presence of potential MR risks such as pacemakers, surgical clips or metallic surgical devices, pregnancy, preventive migraine medications, opiate or hormonal contraceptive use in the 6 months before enrollment, presence of comorbid chronic pain condition, or other neurological or affective disorders. After screening for eligibility, 14 episodic migraineurs and 14 age- and sex-matched HCs were included in this study. We added $11 \mathrm{HC}$ ( 1 male and 10 females) data from another study with the same fMRI protocol ${ }^{23}$ to increase statistical power in the rs-fMRI connectivity analysis.

This study was approved by the University of Michigan institutional review board (HUM00027383) and registered at www.clinicaltrials.gov; identifier: NCT03004313. We obtained written informed consent from all participants involved in the study and conducted the study in compliance with the ethical principles for medical research involving human subjects in the Declaration of Helsinki.

\section{fMRI and PET Data Acquisition}

Figure 1 shows an overview of the data acquisition and analysis flow. We used a $3 \mathrm{~T}$ GE scanner (General Electric, Milwaukee, WI, US) at the University of Michigan for acquiring rs-fMRI and anatomical image data while the patients were outside of migraine attacks. Blood oxygenation level-dependent (BOLD) data were acquired with a reverse spiral sequence ${ }^{24}$ with repetition time $[\mathrm{TR}]=2000 \mathrm{~ms}$, echo time $[\mathrm{TE}]=30 \mathrm{~ms}$, flip angle $=90^{\circ}$, slice thickness $=3.0 \mathrm{~mm}$, field of view $[\mathrm{FOV}]=$ $220 * 220 \mathrm{~mm}$, voxel-size $=3.44 * 3.44 * 3 \mathrm{~mm}$, and acquisition time $=8 \mathrm{~min}$. The participants kept fixating 
A MRI session (interictal visit - 1)

\begin{tabular}{|l|l|l|l|}
\hline-15 & 0 & & 6 \\
SF-MPQ & T1w & & rs-fMRI \\
\hline
\end{tabular}

B PET session (interictal visit - 2)

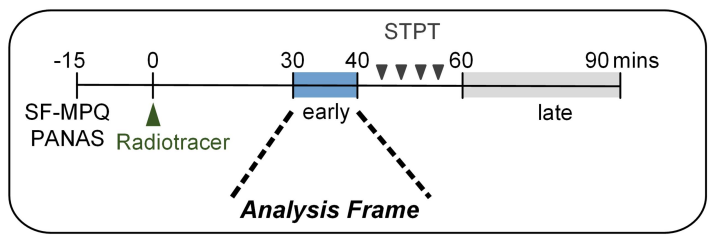

C Clinical Pain (ictal visit)

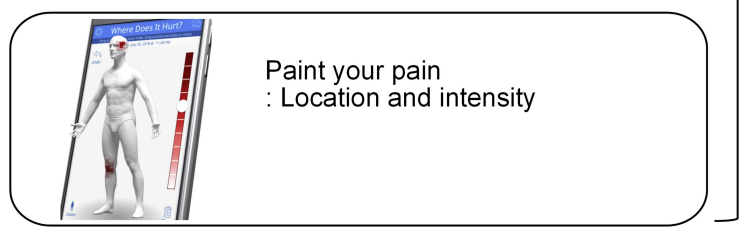

D Associations between D2/3Rs, FC and pain severity

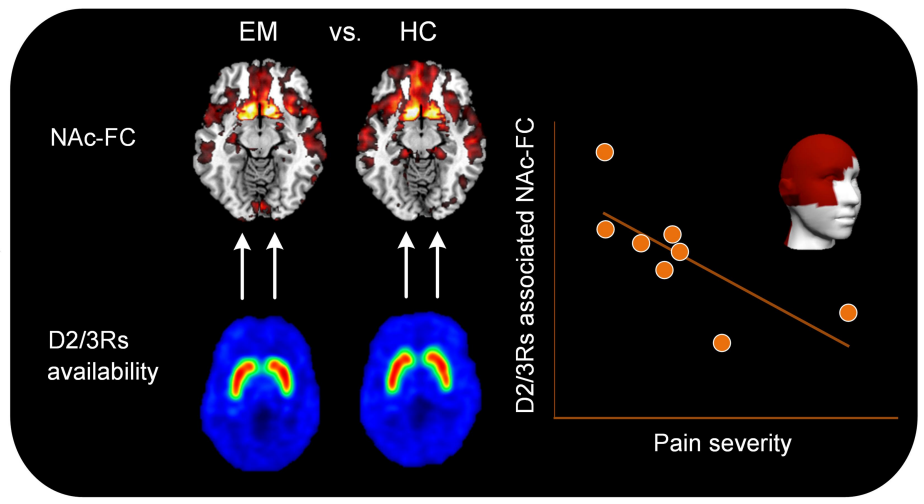

Figure I Data acquisition and analysis flow. We acquired data for (A) magnetic resonance imaging (MRI) and (B) [ " C]raclopride positron emission tomography (PET) from interictal episodic migraine patients and healthy controls. Note that the MRI and PET were acquired on different days. (C) During the ictal PET experiment, patients rated their pain location and intensity using a mobile application (GeoPain, MoxyTech Inc). (D) We first investigated the resting-state functional connectivity for the nucleus accumbens in each side and compared them between episodic migraineurs and healthy controls. Lastly, we tested if the abnormal NAc connectivity would be associated with dopamine D2/D3 receptor availability and clinical pain severity in episodic migraineurs.

their eyes on the cross presented on the computer screen during the fMRI acquisition. We simultaneously acquired the cardiac and respiratory signal with the pulse oximeter (sampling rate $=100 \mathrm{~Hz}$ and $25 \mathrm{~Hz}$, respectively) placed on the subject's finger and the pressure belt placed around each subject's abdomen. Field map imaging was performed with the first volume of the run along with one of the dummy volumes with a different TE $(<2 \mathrm{~ms})$ that are typically discarded upfront. Using the two volumes with different echo times allows us to compute the field map that is used for correction. Thus, aside from the different TE of the one-volume used, all other parameters should be the same as the fMRI data.

Axial fast spoiled gradient recalled echo (SPGR) T1weighted image was acquired with $\mathrm{TR}=12.22 \mathrm{~ms}$, TE = $5.18 \mathrm{~ms}$, flip angle $=15^{\circ}$, slice thickness $=1.0 \mathrm{~mm}$, FOV $=260 * 260 \mathrm{~mm}$, number of excitation $=1$, and voxel size $=1.02 * 1.02 * 1 \mathrm{~mm}$. To reduce head motion, participants used foam padding. We asked participants to stay as still as possible during the whole scan.

PET with a radiotracer $\left[{ }^{11} \mathrm{C}\right]$ raclopride, a selective radiotracer for DA D2/3Rs, was performed with a Simens HR+ scanner (Knoxville, TN) in 3-D mode with septa retracted and scatter correction. Detailed information regarding the administration of the $\left[{ }^{11} \mathrm{C}\right]$ raclopride and reconstruction of individual PET were described elsewhere. ${ }^{25}$ The original 90-minute interictal PET experiment included a resting early-phase (30-40 mins) without stimulation, followed by a sustained thermal pain threshold (STPT) challenge inducing cutaneous allodynia (45-60 mins) and resting late-phase (60-90 mins). Twenty-one frames of PET images were acquired while increasing duration from 30 seconds to 10 minutes. A light forehead restraint was used to prevent head motion. For the entire schedule, the pain specialist confirmed that patients were at least 48-hour free of migraine attacks and abortive medications before the scan time. Also, the patients did not report migraine attacks at least 48-hours after PET/ MRI participation. This study only included the PET early phase analysis without stimulation (30-40 mins) as it matched the MRI protocol (no experimental pain).

In sum, we analyzed fMRI data for 13 EM patients (1 patient was excluded due to a technical issue during the acquisition) and $25 \mathrm{HCs}$. Among them, 11 EM patients and $10 \mathrm{HCs}$ were included in PET analyses.

\section{Resting-State fMRI Preprocessing and Functional Connectivity}

Images were reconstructed using field-map correction and corrected for cardiac- and respiratory-related noise. We utilized the FMRIB Software Library (FSL)(http://www. 
fmrib.ox.ac.uk/fsl) and Analysis of Functional NeuroImages (AFNI)(http://afni.nimh.nih.gov/afni) for pre-processing adapted from 1000 Functional Connectomes Project (http://www.nitrc.org/projects/fcon $1000)$. The pre-processing steps are briefly summarized as follows: discarding the first 5 functional volumes to allow for MR signal equilibration, slice timing correction, 3-dimensional motion correction, grand-mean scaling of the voxel value, removing of eight nuisance signals (six motion parameters, cerebrospinal fluid, and white matter) by regression, removing linear and quadratic trends, and spatial smoothing with a Gaussian kernel (FWHM = $6 \mathrm{~mm})$, and temporal band-pass filtering $(0.01-0.1 \mathrm{~Hz})$. The functional images were then registered to the Montreal Neurological Institute (MNI) $\left(2 * 2 * 2 \mathrm{~mm}^{3}\right)$ standard space using FMRIB's Linear Image Registration Tool. The frame-wise displacement (FD) of time-series was quantified for head motion. ${ }^{26}$ Although the mean FD did not differ between the two groups (EM: $0.054 \pm 0.021$; HC: $0.061 \pm 0.027, \mathrm{t}=0.81, p=0.43$ ), it was added to the statistical group comparison as a covariate to rule out a potential effect of head motion on connectivity measures.

We extracted averaged fMRI time series of left and right NAc seed using the Harvard-Oxford cortical and subcortical structural atlases ${ }^{27}$ to correlate them with each voxel's time series of the whole-brain in native space to create an intrinsic FC map of the NAc. The resulting correlation maps were Fisher's r-to-z converted and then transformed into MNI152 standard space. We conducted a one-sample $t$-test for each group while adjusting age, sex, and mean FD to examine which regions show spatially correlated patterns with the NAc using the DPABI statistical module version 4.2 (http:// rfmri.org/dpabi) ${ }^{28}$ We applied a two-sample $t$-test to identify brain regions with significant differences in each NAcFC between the EM vs $\mathrm{HC}$ while adjusting age, sex, and FD. Multiple comparisons were corrected with a threshold of $p<0.05$ (height threshold of uncorrected $p<0.005$, combined with cluster threshold of $p<0.05$, two-tailed), determined by Monte Carlo simulation $(\mathrm{n}=5000)$ using the DPABI Alphasim program. After that, we extracted the average Fisher's z-score in each significant cluster in which NAc-FC was different between the two groups. In principle, it is desirable to correct statistical maps based on the number of seed points. However, according to the previous literature, ${ }^{29,30}$ left and right NAc are differentially involved in migraine pathophysiology; therefore, we did not correct statistical maps based on the number of seed points.

\section{PET Preprocessing}

We included PET images acquired ranging from 30 to 40minute post tracer administration (early resting-state). They were reconstructed using interactive algorithms into a $128 * 128$ pixel-matrix in a $24 * 24 \mathrm{~cm}$ FOV with attenuation and scatter corrections, as well as head motion artifact correction by performing a linear co-registration through frames of dynamic PET images.

Then, two sets of parametric maps were generated from the reconstructed images, with a voxel-by-voxel basis threshold at $p<0.01$ and an extent threshold of 288 voxels, including 1$)$ a tracer transport measure $\left(\mathrm{K}_{1}\right.$ ratio) usually used for PET-MRI image co-registration and normalization, and 2) a receptor-related measure or a $\mathrm{BP}_{\mathrm{ND}}$ estimated by applying the modified Logan graphical analysis ${ }^{31}$ with the cerebellar gray matter, an area devoid of DA receptors, as a reference region. The slope of the Logan plot is $\left(f_{2} \mathrm{~B}_{\max } / K_{d}\right)$, where $\mathrm{f}_{2}$ is the free fraction of tracer in the non-displacement tissue compartment, $\mathrm{B}_{\max }$ is the density of available binding sites, and $\mathrm{K}_{d}$ is the affinity constant. The uptake measure of the radiotracer $\left[{ }^{11} \mathrm{C}\right]$ raclopride (non-displaceable binding potential, $\mathrm{BP}_{\mathrm{ND}}$ ) was used to assess a DA D2/3Rs availability.

Each $\mathrm{T} 1$ image was linearly aligned to the $\mathrm{K} 1$ ratio image with a mutual information algorithm, ${ }^{32}$ and the resulting matrix was applied to the $\mathrm{BP}_{\mathrm{ND}}$ images. $\mathrm{T} 1$ images were then non-linearly normalized to MNI space using the DARTEL ${ }^{33}$ in SPM8 and Matlab (MathWorks, $\mathrm{R} 2015 \mathrm{~B})$. Both $\mathrm{BP}_{\mathrm{ND}}$ and $\mathrm{K} 1$ ratio images were then normalized into MNI space by applying the resulting deformation parameters. The normalized $\mathrm{BP}_{\mathrm{ND}}$ images were resampled to 2-mm voxels and smoothed with a Gaussian kernel $(3 * 3 * 2 \mathrm{~mm})$. Global normalization was not applied to the parametric map. Only regions of voxel values of $\mathrm{BP}_{\mathrm{ND}}$ over 0.1 times the mean global image was included for analysis. Averaged $\mathrm{BP}_{\mathrm{ND}}$ of each NAc was extracted for each subject using the Harvard-Oxford cortical and subcortical structural atlases. ${ }^{27}$

\section{Pain and Affect Measures}

We acquired demographic and migraine-specific information, including duration, frequency of migraine attacks, and aura at the screening visit. When migraine patients visited for the MRI or PET scanning, we confirmed that they were outside of headache attack and any pain using 
the short-form McGill Pain Questionnaires (SF-MPQ). ${ }^{34}$ Among the patients who participated in both interictal PET and fMRI, 8 patients re-visited for an ictal PET scan and record their pain severity in the craniofacial and cervical region using an in-house developed application called "GeoPain" (MoxyTech Inc, Ann Arbor, MI, https://moxy tech.net/), as described in our previous studies. ${ }^{25,35}$ The GeoPain is a 3D-body navigation system to record objective sensory-discriminative information of pain in realtime, which is quantifiable, reproducible, and validated. ${ }^{36}$ In addition to pain area and average pain intensity by body region (eg, head), it produces a Pain Area and Intensity Number Summation (PAINS) score by multiplying the pain area (from the 220 cells) by their numerical headache intensity (mild=1 (visual analog scale [VAS]: 1-3), moderate $=2$ (VAS: $4-7)$, severe $=3$ (VAS: 8-10)). We divided this individual PAINS score by $660(220 * 3)$ to calculate a percentage estimate of the ictal pain severity.

We applied the Positive and Negative Affect Schedule (PANAS) to assess the present-moment positive and negative affect. ${ }^{37}$ PANAS score of each affect can range from 10-50 with a higher value indicating more positive or negative affect, respectively.

\section{Statistical Analysis Group Comparisons}

Statistical analyses were conducted using STATA software (version 15, StataCorp LP, TX, USA). Demographic, clinical, and PET outcome variables were compared between the two groups (EM vs HC) using an independent samples $t$-test for continuous data or chi-square test for categorical data.

\section{Association Between FC and D2/3Rs Availability}

A general linear model (GLM) was used to test group differences in the association between the NAc-FC strength in each cluster, where the EM group showed an abnormal pattern, and NAc D2/3Rs availability measured by $\left[{ }^{11} \mathrm{C}\right]$ raclopride $\mathrm{BP}_{\mathrm{ND}}$. This model includes each NAc connectivity cluster as a dependent variable and the effects of sex, group, NAc D2/3Rs availability, and group-byNAc D2/3Rs availability interaction term as independent variables. We applied a Bonferroni correction on the resulting $\mathrm{p}$-values $(p<0.05 / 6$ clusters $=0.0083)$. It should be noted that we restricted the association of NAc-FC with NAc D2/3Rs availability on the same side; ie, the right NAc-FC was tested with the right NAc D2/3Rs availability.

\section{Clinical Correlation}

We then evaluated the association between the right NAc connectivity, specifically with the right amygdala, which was differentially modulated by NAc D2/3Rs availability between EM and $\mathrm{HC}$, and interictal affect, and ictal pain severity measured by PAINS. Pearson correlation coefficient was used for associations between those variables. Statistical significance was set as $p<0.05$. We performed additional correlation analyses between affect and pain measures. Also, we examined the association between interictal NAc D2/3Rs availability and positive or negative affect scores in each group.

\section{Results}

\section{Sample Characteristics}

The interictal EM patients and control subjects did not significantly differ in demographic variables, including age and sex composition and PANAS positive and negative affect scores. Table 1 provides a summary of descriptive statistics for each variable and between-group comparison results.

\section{Group Difference in Brain Measures}

We first ascertained that the NAc has a spontaneous rs-FC with regions, including the prefrontal cortex, ACC, thalamus, insula, amygdala, and hippocampus, constituting the previously known as the mesolimbic reward circuitry in both groups (Supplementary Figure 1). The two-sample $t$-test showed that, relative to controls, the EM group showed weaker right NAc-FC with the right amygdala (peak-xyz: $30.4,-20$, peak-t $=-4.3$, voxels $=49$ ), right hippocampus (peak-xyz: 26,-36,4, peak-t $=-4.2$, voxels $=51$ ), right thalamus (peak-xyz: $22,-28,6$, peak-t $=-4.1$, voxels $=46$ ), and left rostral ACC (peak-xyz: -8,40,2, peak- $\mathrm{t}=3.5$, voxels $=40)($ Figure $2 \mathrm{~A})$. Conversely, the EM group showed stronger left NAc-FC with the left dorsolateral prefrontal (DLPFC)(peak-xyz: -32,44,34, peak- $\mathrm{t}=4.0$, voxels $=38$ ), and right lingual gyrus (peakxyz: $16,-102,0$, peak-t $=4.2$, voxels $=71$ ) than the $\mathrm{HC}$ group (Figure $2 \mathrm{~B}$ ). When we compared the NAc $\left[{ }^{11} \mathrm{C}\right]$ raclopride $\mathrm{BP}_{\mathrm{ND}}$ (DA D2/3Rs availability) between the interictal $\mathrm{EM}$ and $\mathrm{HCs}$, there were not any differences between the two groups (Right NAc: $\mathrm{t}=-0.47, p=0.69$; Left NAc: $\mathrm{t}=-0.25, \mathrm{p}=0.81$ ). This result was not changed after adding age or sex as covariates in a linear regression model. 
Table I Demographic and Clinical Characteristics of Participants

\begin{tabular}{|c|c|c|c|}
\hline & Episodic Migraine & Healthy Control & Comparisons \\
\hline \multicolumn{4}{|l|}{ Screening visit } \\
\hline No. (F:M) & $13(7: 6)$ & $25(18: 7)$ & $\chi^{2}=1.25, p=0.26$ \\
\hline Age, year & $27.8 \pm 6.1$ & $26.7 \pm 7.4$ & $t=0.47, p=0.64$ \\
\hline Chronicity, year & $12.6 \pm 7.0$ & NA & NA \\
\hline Frequency/month, no. & $5.8 \pm 2.9$ & NA & NA \\
\hline Aura, with: without & $9: 4$ & NA & NA \\
\hline \multicolumn{4}{|l|}{ Visit at interictal phase } \\
\hline No. (F:M) & II (6:5) & $10(5: 5)$ & $\chi^{2}=0.04, p=0.84$ \\
\hline Age, year & $28.0 \pm 6.3$ & $24.3 \pm 6.1$ & $t=1.37, p=0.19$ \\
\hline PANAS positive & $26.0 \pm 7.5$ & $27.7 \pm 8.5$ & $\mathrm{t}=-0.5 \mathrm{I}, \mathrm{p}=0.62$ \\
\hline PANAS negative & $13.3 \pm 4.5$ & $13.0 \pm 4.0$ & $t=0.17, p=0.87$ \\
\hline \multicolumn{4}{|l|}{ Visit at ictal phase } \\
\hline No. (F:M) & $8(4: 4)$ & NA & NA \\
\hline Pain severity by PAINS , \% & $|4.5| \pm 9.12$ & NA & NA \\
\hline Pain area $(0-220)$ & $40.5 \pm 21.33$ & NA & NA \\
\hline Pain intensity $(\mathrm{I}-3)^{\mathrm{b}}$ & $2.37 \pm 0.51$ & NA & NA \\
\hline
\end{tabular}

Notes: Values are mean \pm standard deviation otherwise indicated. ${ }^{\text {T}}$ The patients during the ictal phase used GeoPain mobile application to record the pain intensity and area

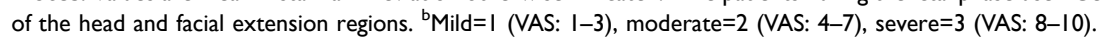

Abbreviations: NA, not applicable; PAINS, pain area and intensity number summation; PANAS, positive and negative affect schedule; VAS, visual analog scale.

\section{Association of Altered NAc Connectivity with DA D2/3Rs Availability}

To identify whether the altered NAc connectivity observed in the EM group was associated with NAc DA D2/3Rs availability, we performed the GLM analysis to examine an effect of the group-by-DA D2/3Rs availability on each cluster. A significant interaction effect was found only in the right NAc connectivity with the right amygdala $(\mathrm{z}=-2.72, \mathrm{p}=0.007)$, but not in other clusters; left rostral ACC $(\mathrm{z}=-0.53, \mathrm{p}=$ $0.59)$, right thalamus $(z=-1.17, p=0.24)$, right hippocampus $(\mathrm{z}=0.10, \mathrm{p}=0.92)$, left DLPFC $(\mathrm{z}=-0.36, \mathrm{p}=$ $0.72)$, and right lingual gyrus $(z=0.26, p=0.80)$. This significant result was driven by a negative association between right NAc D2/3Rs availability and right NAcamygdala connectivity in the migraine group. There was a trend-level positive association between those two measures in the control group (Figure 3A).

\section{Association of NAc-Amygdala}

\section{Connectivity with Affect and Pain}

We assessed whether right NAc-amygdala connectivity, which was negatively modulated by NAc D2/3Rs availability, was related to affect level and pain severity. As a result, patients exhibiting weaker right NAc-amygdala connectivity had lower interictal positive affect $(r=0.77$, $\mathrm{p}=0.006$ ) (Figure 3B). But, this was not true for negative affect $(r=0.22, p=0.51)$. Also, there was a strong inverse relationship between right NAc D2/3Rs availability and positive affect only in the EM group (Figure 3B). However, we did not find a significant relationship between right NAc D2/3Rs availability and negative affect in both groups (all $p$ 's $>0.45$ ). Neither positive nor negative affect scores were correlated with left NAc D2/3Rs availability in both groups (all $p$ 's $>0.08$ ). Crucially, the right NAc- amygdala connectivity was inversely correlated with pain severity over the head and facial extension area $(\mathrm{r}=-0.75, \mathrm{p}=0.033, \mathrm{n}=8)$ measured during a separate visit for the ictal PET experiment (Figure 3C). We additionally found that patients with lower interictal positive affect exhibit greater ictal pain severity $(r=-0.71, p=$ 0.050 ). But, this was not true for the relationship between negative affect and ictal pain severity $(r=0.24, p=0.56)$.

\section{Discussion}

This study mainly explored whether NAc connectivity, as a functional measure of reward network, is related to endogenous DA D2/3Rs signaling and sought to reveal 
A Group difference of R-NAc FC

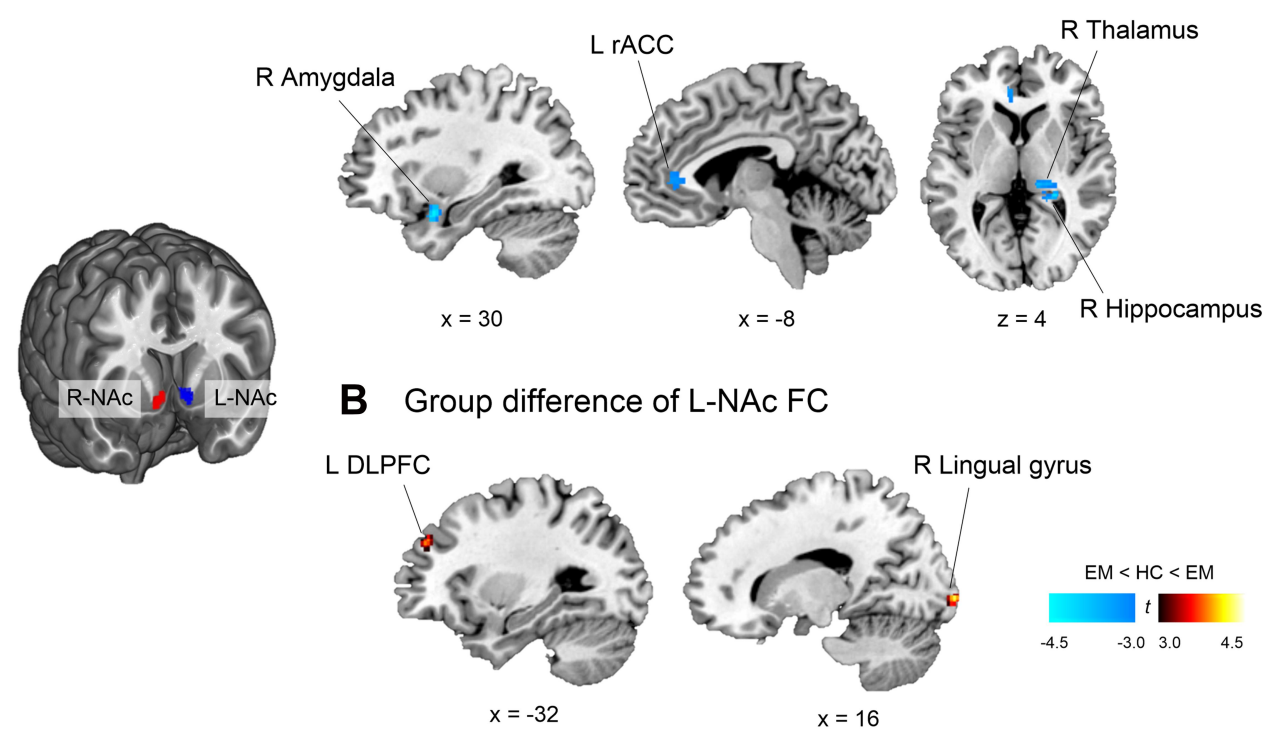

Figure 2 Between-group comparison of whole-brain wise resting-state functional connectivity with the right and left nucleus accumbens. The statistical map was corrected with a threshold of $p<0.05$ (height threshold of uncorrected $p<0.005$, combined with a cluster extent threshold of $p<0.05$ ). Brain slices are presented based on MNI coordinates. (A) Compared with HC, patients with EM showed significantly lower R-NAc FC in the right amygdala, hippocampus, thalamus, and left rACC. (B) They also showed significantly higher L-NAc FC in the left DLPFC and right lingual gyrus.

Abbreviations: EM, episodic migraine; DLPFC, dorsolateral prefrontal cortex; HC, healthy control; NAc, nucleus accumbens; rACC, rostral anterior cingulate cortex.

its clinical significance in EM. We found that lower right NAc-amygdala connectivity was associated with higher NAc D2/3Rs availability indicating a more upregulated DA D2/3Rs or less DA neurotransmission. Notably, patients showing weaker right NAc-amygdala connectivity exhibit lower interictal positive affect and greater ictal pain severity over the craniofacial area. These results support our hypothesis that the endogenous DA D2/3Rs availability interacts with the mesolimbic reward circuitry in a way that regulates positive affect and ictal pain severity in migraine and agrees with the previous hypothesis that the hedonic continuum with pain and reward depends on the dopaminergic reward system. ${ }^{38,39}$

Regarding the NAc connectivity results, episodic migraineurs showed lower right NAc connectivity with the reward circuitry's key components, including the right amygdala, hippocampus, thalamus, and left rostral ACC, compared to the HC group, suggesting that the reward pathway in episodic migraine is less integrated or functionally communicating. ${ }^{40}$ Based on our finding that patients having lower right NAc FC with the right amygdala exhibited lower positive affect and higher pain severity, less integrated right NAc FC would, at least in part, reflect an abnormal affective processing or emotional response to pain. However, in the study by Yuan et al (2013), ${ }^{29}$ compared with HC, EM patients without aura
(MoWA) exhibited higher right NAc connectivity with orbitofrontal gyrus, parahippocampal gyrus, and bilateral ACC whose location partially overlapped with our result showing a lower right NAc FC with the left rostral ACC in EM than HC. In the same study, stronger right NAc-ACC connectivity was correlated with the migraine's chronicity, roughly ranging from 2 to 32 years. Notably, their chronicity range is $60 \%$ longer than that of our patients, which runs between 2-20 years, pointing to a possibility that chronicity might account for the differences between the two studies. Additionally, the presence of aura might underlie the different results between the studies, ${ }^{41}$ considering that we included both migraines with aura (MWA) and MoWA. There is still an insufficient number of studies investigating resting-state migrainous brain between MoWA and MWA. However, these findings indicated that visual ${ }^{42,43}$ and default-mode network ${ }^{44}$ are most likely implicated in the differences observed in brain activity between the two migraine subtypes.

Interestingly, the recent study by Schulte et al $(2020),{ }^{30}$ in which 9 EM patients underwent daily rsfMRI for a minimum period of 30 consecutive days, demonstrated that the right NAc, but not left side, connectivity with the left amygdala, hippocampus, and parahippocampal gyrus was stronger during the preictal phase ( 24 hrs before headache onset) than the interictal 
A Association of NAc-FC with DA D2/3Rs availability

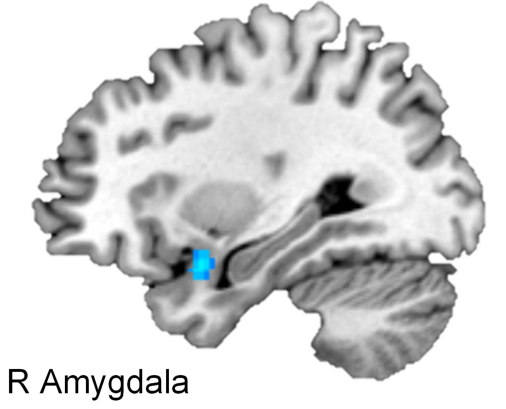

$x=30$

- EM $r=-0.64, p=0.036, n=11$

- $\mathrm{HC} \quad \mathrm{r}=0.63, p=0.053, \mathrm{n}=10$

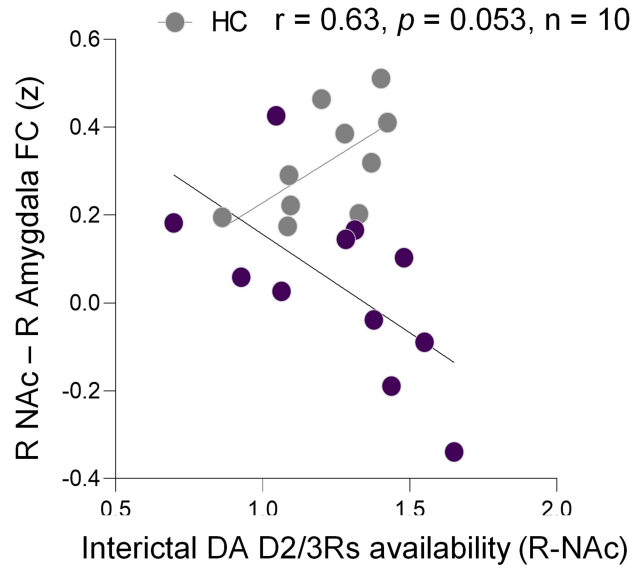

B - Association of NAc-FC and DA D2/3Rs availability with affect -
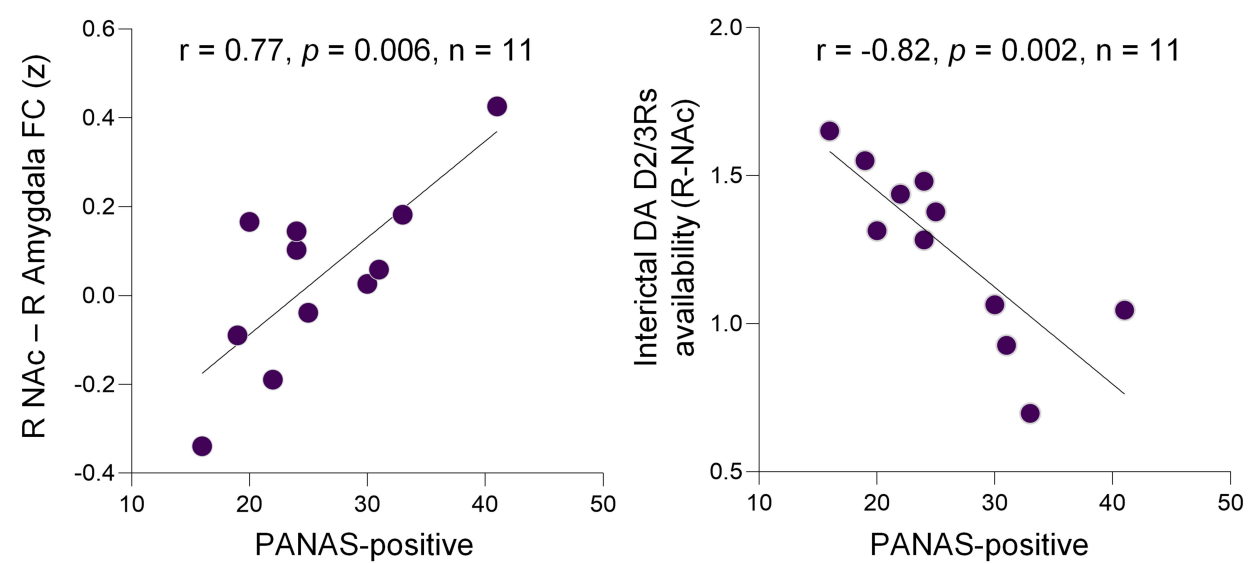

C

Association of NAc-FC with ictal pain

Pain area and intensity number summation (PAINS)

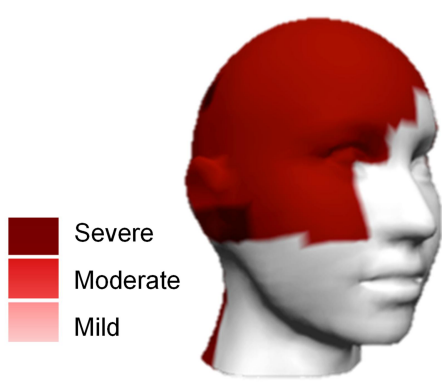

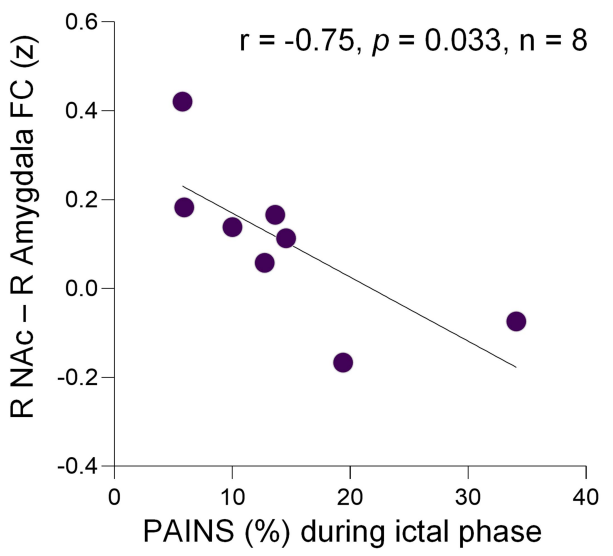

Figure 3 Relationship between the right NAc-amygdala connectivity and NAc D2/3Rs availability, positive affect, and pain severity. (A) Less right NAc-amygdala connectivity was coupled with higher right NAc D2/3Rs availability in the EM group, whereas this relationship was opposite for the HC group. (B) Patients with lower interictal positive affect had weaker NAc-amygdala connectivity and higher right NAc D2/3Rs availability. (C) Weaker interictal NAc-amygdala connectivity was associated with greater pain severity measured by PAINS during their ictal phase. Averaged pain intensity and area for 8 patients measured by the GeoPain mobile application were schematically represented on the $3 \mathrm{D}$ head image at the lower left.

phase. This study indicated that the right NAc connectivity depends on the time-lapse from a headache attack and is further implicated in the generation of migraine attacks. 
In contrast, the regions showing enhanced connectivity with the left NAc included the left dorsolateral prefrontal cortex (DLPFC) and right lingual gyrus. The DLPFC and nucleus accumbens constitute the cortico-striatal circuit and perform top-down control of pain sensitivity and emotion. ${ }^{45}$ Given that the EM group had no pain or migraine attack around the time of fMRI acquisition, it might reflect an adaptive neural strategy for pain modulation during the interictal phase.

We confirmed that healthy controls had no statistically significant FC between the left NAc and right lingual gyrus (Supplemental Figure 1). However, EM patients exhibited positive FC between the two regions, which should be considered atypical. The growing amount of literature suggests that the hyper-excitable or hyperconnected lingual gyrus has been implicated with visual disturbances of migraineurs such as blurred vision, photophobia, or visual aura. ${ }^{42,46}$ For example, resting-state FC between the right lingual gyrus and cerebellum was linked to visual light sensitivity in MwoA. ${ }^{46}$ Interestingly, dopaminergic reward network has been hypothesized mechanisms underlying the auditory and visual hallucination of schizophrenia. ${ }^{47}$ Moreover, NAc activation was related to its vividness. ${ }^{48}$ Together, we cautiously speculate that the left NAc hyper-connected to the right lingual gyrus may indicate an atypical visual experience related to migraine attack or headache severity. However, further studies are needed to confirm these hypotheses.

Previous FC studies are heterogeneous in terms of clinical characteristics, such as aura, attack frequency, medication, and the analytic method to quantify a functional network. These factors did not allow us to directly compare previous results with the current one, as noted in ${ }^{49}$ thus, future studies should assess in detail the impact of each of those clinical characteristics in migraine.

Although our study was not designed to explain whether altered NAc connectivities were acquired or vulnerable traits, chronic emotional/physiological stress (eg, repetitive migraine attacks) could change functional networks in a maladaptive way. For example, in an investigation of hippocampal connectivity in response to noxious heat stimuli, EM patients with high-frequency attacks (8-14 days/month) showed less connectivity with pain processing areas, including the bilateral NAc, than patients with low-frequency attacks (1-2 days/month). ${ }^{50}$ However, the current study can not rule out a possibility that alterations in NAc connectivity with the amygdala, hippocampus, and DLPFC would reflect a vulnerability to the development or maintenance of migraine. A previous longitudinal neuroimaging study with chronic back pain (CBP) provided compelling evidence that structural and functional corticolimbic alterations were a predetermining factor of developing chronic pain. ${ }^{51}$ Briefly, patients who developed from subacute to persistent CBP exhibited higher connectivity between the medial PFC, amygdala, and NAc, relative to those who recovered from subacute pain and healthy participants.

Next, we explored whether altered NAc connectivity was influenced by DA D2/3Rs availability. As expected, we found that only the right NAc-amygdala connectivity was negatively correlated with the right NAc D2/3Rs availability in the EM group. This relationship was inverted, which was marginally significant in the control group. That direction of association is the same as the previous study with major depressive disorder reporting an association between higher $\mathrm{BP}_{\mathrm{ND}}$ (reduced striatal $\mathrm{DA}$ release) and lower striatal connectivity with the defaultmode and salience networks. ${ }^{20}$

Although DA deficiency or hypo-functionality has been recognized as a part of the migraine pathophysiology, ${ }^{52}$ we did not find a significant difference between the interictal EM and $\mathrm{HC}$ for the NAc D2/3Rs availability. However, previous studies with CBP and fibromyalgia reported reduced DA receptor availability of the striatal regions at baseline. ${ }^{14,53}$ These discrepancies among studies could be explained by pain origins (neuropathies vs musculoskeletal), ${ }^{54}$ presence of current pain, or regional specificity (striatal vs extra-striatal). We reasoned that sufficient pain relief, possibly interpreted as rewarding to interictal migraine patients, might compensate for less DA neurotransmission or upregulated DA receptors.

We also found that interictal positive affect was negatively correlated with right NAc D2/3Rs availability and positively with the right NAc-amygdala connectivity. This result is partly understood under the dopaminergic theory of positive affect, which links positive affect and DA levels. ${ }^{55}$ However, our result did not occur simply due to positive affect since this relationship was observed only in the patient group. Alternatively, we thought that the result is likely related to an individual trait, such as reward responsiveness, positively correlated with PANAS positive affect. ${ }^{56}$ The patients might perceive the interictal state as being rewarded because the relief-period of an aversive event (pain) can be a substantial reward. ${ }^{57} \mathrm{We}$, therefore, speculated that an inter-individual difference of how 
rewarded or motivated by pain relief would interact with the DA D2/3Rs system.

It is well established that the amygdala is responsible for the emotional-affective processing of pain. ${ }^{58}$ The basolateral nuclei of the amygdala, showing lower connectivity with the NAc in EM patients, is involved in associative learning of emotional valance, ${ }^{59}$ anxiety, ${ }^{60}$ threat modulation, ${ }^{61}$ and negative valence of pain. ${ }^{62}$ Notably, Martikainen et al (2015) ${ }^{14}$ demonstrated a positive association between DA D2/3Rs availability of the right NAc and $\mu$-opioid receptor (MOR) availability of the bilateral amygdala in CBP patients. As stated by the authors, considering the reciprocal interaction between opioid and DA system, MOR function of the amygdala might be compromised as the NAc's D2/3Rs function of the NAc deteriorates in chronic pain. Taken together, our findings of lower NAc-basolateral amygdala connectivity could reflect a degraded interaction between DA and opioid system, which further links reward and pain modulatory systems in migraine patients.

As an additional result, we found that interictal positive affect was negatively correlated with ictal pain severity. This result is supported by a meta-analysis study demonstrating an inverse relationship between positive affect and pain severity in people with chronic non-cancer pain. ${ }^{63} \mathrm{We}$ think that the NAc-amygdala network, which underpins positive emotional valence, ${ }^{64}$ might play a role in buffering or facilitating clinical pain. However, how the dopaminergic neurotransmission through the reward circuitry influences migraine pain requires further investigation. From a behavioral perspective, when patients experience rewards in a pain-free state, it possibly affects motivation and learning through dopaminergic reward circuitry. It might then influence patients to modify their behavior to prevent future attacks or to mitigate pain intensity. ${ }^{65}$ Thus, this finding also corroborates the importance of cognitivebehavioral therapies in migraine treatment. ${ }^{66,67}$

The multimodal study, with fMRI and PET, provided insight into understanding the reward system's involvement in pain and affect in migraine. Our PET findings and complex protocols (eg, non-induced attacks, same menstrual phase) added crucial molecular information behind the MRIbased connectivity dysfunction. Due to the study design's complexities, it might be understandable to first conduct a correlation analysis between FC and pain severity only in a highly scrutinized number of patients who visited both interictal and ictal phases. Nonetheless, we investigated clinical symptoms and functional brain signals in a timely manner (not dependent on patients' memory about pain and affect, which might be erroneous or biased), which brings more reliability to our findings. Still, they should be later extended to a larger sample size. For the same reason, we could not analyze the difference between migraine subtype (eg, aura) and sex, which would likely affect the current results. On a related note, given the exploratory nature of our analysis, we did not perform statistical corrections for multiple clinical correlations. ${ }^{68}$ Lastly, medication should be taken into account. Although we excluded participants who used to take opioids, psychostimulant drugs, or preventive medicine and ask patients to stop medications except for non-steroidal anti-inflammatory drugs (NSAIDs) at least 48hrs before scan time, their medication history may have interacted with the brain's chemical and regional connectivities differently among the patients. Thus, it needs a particular caution while interpreting our results.

\section{Conclusion}

In sum, this study provides evidence of the association between the mesolimbic reward connectivity and DA D2/ 3Rs availability and its impact on clinical manifestations in EM. Notably, the NAc-amygdala connectivity associated with dopaminergic neurotransmission would encode an emotional valence, ictal pain severity (area/intensity), and possibly the relationship between positive affect and pain. Our findings suggest the system-level mechanism underlying pain and affect in migraine that could shed light on innovative and improved treatment strategies, such as enhancing positive affect or modulating reward circuitry for alleviating migraine pain severity.

\section{Abbreviations}

$\mathrm{ACC}$, anterior cingulate cortex; $\mathrm{BP}_{\mathrm{ND}}$, non-displaceable binding potential; DA, dopamine; D2/3Rs, D2/D3 receptors; EM, episodic migraine; FC, functional connectivity; FD, frame-wise displacement; $\mathrm{HC}$, healthy control; NAc, nucleus accumbens; PANAS, positive and negative affect schedule; PAINS, pain area and intensity number summation; PET, positron emission tomography; rs-fMRI, resting-state functional magnetic resonance imaging.

\section{Data Sharing Statement}

The data supporting the findings of this study are available from the corresponding author upon reasonable request made by email. Upon an agreement to share, the de-identified data will be accessed via a secured portal provided by the 
University of Michigan for 2 years after study completion (2023 Feb).

\section{Acknowledgment}

We thank Jacqueline Dobson for her help in proofreading the paper.

\section{Funding}

This study was supported by grants from the National Institutes of Health - National Institute of Neurological Disorders and Stroke (NIH-NINDS K23 NS062946, R01 NS094413) and NIH-National Institute of Dental and Craniofacial Research (NIDCR) (U01-DE025633) USA (A.D); the Dana Foundation's Brain and ImmunoImaging Award USA (A.D); and the Migraine Research Foundation Research Grant Award, USA (A.D). This study is not industry-sponsored.

\section{Disclosure}

Dr. Alexandre F. DaSilva co-created GeoPain, used in the current study for measuring pain severity, and also cofounded and co-owned the start-up MoxyTech, Inc. (Ann Arbor, MI) that licensed the technology from the University of Michigan. Dr. DaSilva also reports patent application issued to GeoPain. The authors declare no other potential conflicts of interest regarding the authorship and/or publication of this article.

\section{References}

1. Charles A. The pathophysiology of migraine: implications for clinical management. Lancet Neurol. 2018;17(2):174-182. doi:10.1016/ S1474-4422(17)30435-0

2. Bigal ME, Ashina S, Burstein R, et al. Prevalence and characteristics of allodynia in headache sufferers: a population study. Neurology. 2008;70(17):1525-1533. doi:10.1212/01.wnl.0000310645.31020.b1

3. Lantéri-Minet M, Radat F, Chautard M-H, Lucas C. Anxiety and depression associated with migraine: influence on migraine subjects' disability and quality of life, and acute migraine management. Pain. 2005;118(3):319-326. doi:10.1016/j.pain.2005.09.010

4. Borsook D, Maleki N, Becerra L, McEwen B. Understanding migraine through the lens of maladaptive stress responses: a model disease of allostatic load. Neuron. 2012;73(2):219-234. doi:10.1016/j. neuron.2012.01.001

5. Kocsel N, Galambos A, Szabó E, et al. Altered neural activity to monetary reward/loss processing in episodic migraine. Sci Rep. 2019;9(1):5420. doi:10.1038/s41598-019-41867-x

6. DosSantos MF, Moura BDS, DaSilva AF. Moura B de S, DaSilva AF. Reward circuitry plasticity in pain perception and modulation. Front Pharmacol. 2017;8:790. doi:10.3389/fphar.2017.00790

7. Cahill CM, Cook C, Pickens S. Migraine and reward system-or is it aversive? Curr Pain Headache Rep. 2014;18(5):410. doi:10.1007/ s11916-014-0410-y
8. Serafini RA, Pryce KD, Zachariou V. The Mesolimbic Dopamine System in Chronic Pain and Associated Affective Comorbidities. Biol Psychiatry. 2020;87(1):64-73. doi:10.1016/j.biopsych.2019.10.018

9. Hikida T, Morita M, Macpherson T. Neural mechanisms of the nucleus accumbens circuit in reward and aversive learning. Neurosci Res. 2016;108:1-5. doi:10.1016/j.neures.2016.01.004

10. Scott DJ, Heitzeg MM, Koeppe RA, Stohler CS, Zubieta J-K. Variations in the human pain stress experience mediated by ventral and dorsal basal ganglia dopamine activity. $J$ Neurosci. 2006;26 (42):10789-10795. doi:10.1523/JNEUROSCI.2577-06.2006

11. Scott DJ, Stohler CS, Egnatuk CM, Wang H, Koeppe RA, Zubieta J-K. Placebo and nocebo effects are defined by opposite opioid and dopaminergic responses. Arch Gen Psychiatry. 2008;65 (2):220-231. doi:10.1001/archgenpsychiatry.2007.34

12. Navratilova E, Xie JY, Okun A, et al. Pain relief produces negative reinforcement through activation of mesolimbic reward-valuation circuitry. Proc Natl Acad Sci U S A. 2012;109(50):20709-20713. doi:10.1073/pnas.1214605109

13. Porreca F, Navratilova E. Reward, motivation, and emotion of pain and its relief. Pain. 2017;158(Suppl):S43-S49. doi:10.1097/j. pain. 0000000000000798

14. Martikainen IK, Nuechterlein EB, Peciña M, et al. Chronic back pain is associated with alterations in dopamine neurotransmission in the ventral striatum. $J$ Neurosci. 2015;35(27):9957-9965. doi:10.1523/ JNEUROSCI.4605-14.2015

15. Baliki MN, Petre B, Torbey S, et al. Corticostriatal functional connectivity predicts transition to chronic back pain. Nat Neurosci. 2012;15(8):1117-1119. doi:10.1038/nn.3153

16. Harris HN, Peng YB. Evidence and explanation for the involvement of the nucleus accumbens in pain processing. Neural Regen Res. 2020;15(4):597-605. doi:10.4103/1673-5374.266909

17. Dang LC, O'Neil JP, Jagust WJ. Dopamine supports coupling of attention-related networks. $J$ Neurosci. 2012;32:9582-9587. doi:10.1523/JNEUROSCI.0909-12.2012

18. Shafiei G, Zeighami Y, Clark CA, et al. Dopamine signaling nodulates the stability and integration of intrinsic brain networks. Cereb Cortex. 2019;29(1):397-409. doi:10.1093/cercor/bhy264

19. Alavash M, Lim SJ, Thiel C, Sehm B, Deserno L, Obleser J. Dopaminergic modulation of hemodynamic signal variability and the functional connectome during cognitive performance. Neuroimage. 2018;172:341-356. doi:10.1016/j.neuroimage.2018. 01.048

20. Hamilton JP, Sacchet MD, Hjørnevik T, et al. Striatal dopamine deficits predict reductions in striatal functional connectivity in major depression: a concurrent $11 \mathrm{C}$-raclopride positron emission tomography and functional magnetic resonance imaging investigation. Transl Psychiatry. 2018;8(1):264. doi:10.1038/ s41398-018-0316-2

21. McCutcheon RA, Nour MM, Dahoun T, et al. Mesolimbic Dopamine Function Is Related to Salience Network Connectivity: an Integrative Positron Emission Tomography and Magnetic Resonance Study. Biol Psychiatry. 2019;85(5):368-378. doi:10.1016/j.biopsych.2018.09.010

22. Headache Classification Committee of the International Headache Society. The International Classification of Headache Disorders, 3rd edition (beta version). Cephalalgia. 2013;33(9):629-808. doi: $10.1177 / 0333102413485658$

23. Nascimento TD, Yang N, Salman D, et al. $\mu$-opioid activity in chronic TMD pain is associated with COMT polymorphism. $J$ Dent Res. 2019;98(12):1324-1331. doi:10.1177/0022034519871938

24. Glover GH, Law CS. Spiral-in/out BOLD fMRI for increased SNR and reduced susceptibility artifacts. Magn Reson Med. 2001;46 (3):515-522. doi:10.1002/mrm.1222

25. DaSilva AF, Nascimento TD, Jassar H, et al. Dopamine D2/D3 imbalance during migraine attack and allodynia in vivo. Neurology. 2017;88(17):1634-1641. doi:10.1212/WNL.0000000000003861 
26. Power JD, Barnes KA, Snyder AZ, Schlaggar BL, Petersen SE. Spurious but systematic correlations in functional connectivity MRI networks arise from subject motion. Neuroimage. 2012;59:2142-2154. doi:10.1016/j.neuroimage.2011.10.018

27. Toro R, Fox PT, Paus T. Functional coactivation map of the human brain. Cereb Cortex. 2008;18(11):2553-2559. doi:10.1093/cercor/ bhn014

28. Yan C-G, Wang X-D, Zuo X-N, Zang Y-F. DPABI: data processing \& analysis for (resting-State) brain imaging. Neuroinformatics. 2016;14 (3):339-351. doi:10.1007/s12021-016-9299-4

29. Yuan K, Zhao L, Cheng P, et al. Altered structure and resting-state functional connectivity of the basal ganglia in migraine patients without aura. J Pain. 2013;14(8):836-844. doi:10.1016/j. jpain.2013.02.010

30. Schulte LH, Menz MM, Haaker J, May A. The migraineur's brain networks: continuous resting state fMRI over 30 days. Cephalalgia. 2020;40(14):1614-1621. doi:10.1177/0333102420951465

31. Logan J, Fowler JS, Volkow ND, Wang G-J, Ding Y-S, Alexoff DL. Distribution volume ratios without blood sampling from graphical analysis of pet data. J Cereb Blood Flow Metab. 1996;16 (5):834-840. doi:10.1097/00004647-199609000-00008

32. Meyer CR, Boes JL, Kim B, et al. Demonstration of accuracy and clinical versatility of mutual information for automatic multimodality image fusion using affine and thin-plate spline warped geometric deformations. Med Image Anal. 1997;1(3):195-206. doi:10.1016/ S1361-8415(97)85010-4

33. Ashburner J. A fast diffeomorphic image registration algorithm. Neuroimage. 2007;38(1):95-113. doi:10.1016/j.neuroimage.2007.07.007

34. Melzack R. The short-form McGill pain questionnaire. Pain. 1987;30 (2):191-197. doi:10.1016/0304-3959(87)91074-8

35. Jassar H, Nascimento TD, Kaciroti N, et al. Impact of chronic migraine attacks and their severity on the endogenous $\mu$-opioid neurotransmission in the limbic system. NeuroImage Clin. 2019;23:101905. doi:10.1016/j.nicl.2019.101905

36. Kaciroti N, DosSantos MF, Moura B, et al. Sensory-Discriminative three-dimensional body pain mobile app measures versus traditional pain measurement with a visual analog scale: validation study. JMIR mHealth uHealth. 2020;8:e17754. doi:10.2196/17754

37. Watson D, Clark LA, Tellegen A. Development and validation of brief measures of positive and negative affect: the PANAS scales. $J$ Pers Soc Psychol. 1988;54(6):1063-1070. doi:10.1037/00223514.54.6.1063

38. Becker S, Navratilova E, Nees F, Van Damme S. Emotional and motivational pain processing: current state of knowledge and perspectives in translational research. Pain Res Manag. 2018;2018:1-12. doi:10.1155/2018/9305026

39. Leknes S, Tracey I. A common neurobiology for pain and pleasure. Nat Rev Neurosci. 2008;9(4):314-320. doi:10.1038/nrn2333

40. Cohen AL, Fair DA, Dosenbach NUF, et al. Defining functional areas in individual human brains using resting functional connectivity MRI. Neuroimage. 2008;41(1):45-57. doi:10.1016/j.neuroimage.2008.01.066

41. Kincses ZT, Veréb D, Faragó P, et al. Are migraine with and without aura really different entities? Front. Neurol. 2019.

42. Tedeschi G, Russo A, Conte F, et al. Increased interictal visual network connectivity in patients with migraine with aura. Cephalalgia. 2016;36(2):139-147. doi:10.1177/0333102415584360

43. Niddam DM, Lai KL, Fuh JL, Chuang CYN, Chen WT, Wang SJ. Reduced functional connectivity between salience and visual networks in migraine with aura. Cephalalgia. 2016;36(1):53-66. doi: $10.1177 / 0333102415583144$

44. Lo Buono V, Bonanno L, Corallo F, et al. Functional connectivity and cognitive impairment in migraine with and without aura. $J$ Headache Pain. 2017;18(1):72. doi:10.1186/s10194-017-0782-6

45. Martinez E, Lin HH, Zhou H, Dale J, Liu K, Wang J. Corticostriatal regulation of acute pain. Front Cell Neurosci. 2017;11. doi:10.3389/ fncel.2017.00146
46. Zhang D, Huang X, Su W, et al. Altered lateral geniculate nucleus functional connectivity in migraine without aura: a resting-state functional MRI study. J Headache Pain. 2020;21(1). doi:10.1186/s10194020-01086-6

47. Rolland B, Amad A, Poulet E, et al. Resting-state functional connectivity of the nucleus accumbens in auditory and visual hallucinations in schizophrenia. Schizophr Bull. 2015;41(1):291-299. doi:10.1093/schbul/sbu097

48. Raij TT, Valkonen-Korhonen M, Holi M, Therman S, Lehtonen J, Hari R. Reality of auditory verbal hallucinations. Brain. 2009.

49. Skorobogatykh K, van Hoogstraten WS, Degan D, et al. Functional connectivity studies in migraine: what have we learned? J Headache Pain. 2019;20(1):108. doi:10.1186/s10194-019-1047-3

50. Maleki N, Becerra L, Brawn J, McEwen B, Burstein R, Borsook D. Common hippocampal structural and functional changes in migraine. Brain Struct Funct. 2013;218(4):903-912. doi:10.1007/s00429-0120437-y

51. Vachon-Presseau E, Tétreault P, Petre B, et al. Corticolimbic anatomical characteristics predetermine risk for chronic pain. Brain. 2016;139(7):1958-1970. doi:10.1093/brain/aww100

52. Barbanti P, Fofi L, Aurilia C, Egeo G. Dopaminergic symptoms in migraine. Neurol Sci. 2013;34(S1):67-70. doi:10.1007/s10072-013$1415-8$

53. Wood PB, Schweinhardt P, Jaeger E, et al. Fibromyalgia patients show an abnormal dopamine response to pain. Eur $J$ Neurosci. 2007;25(12):3576-3582. doi:10.1111/j.1460-9568.2007.05623.x

54. Martikainen IK, Hagelberg N, Jääskeläinen SK, Hietala J, Pertovaara A. Dopaminergic and serotonergic mechanisms in the modulation of pain: in vivo studies in human brain. Eur J Pharmacol. 2018;834:337-345. doi:10.1016/j.ejphar.2018.07.038

55. Ashby FG, Isen AM, Turken AU. A neuropsychological theory of positive affect and its influence on cognition. Psychol Rev. 1999;106 (3):529-550. doi:10.1037/0033-295X.106.3.529

56. Ivo VDB, Franken IHA, Muris P. A new scale for measuring reward responsiveness. Front Psychol. 2010;1:239. doi:10.3389/ fpsyg. 2010.00239

57. Navratilova E, Atcherley CW, Porreca F. Brain Circuits Encoding Reward from Pain Relief. Trends Neurosci. 2015;38(11):p. 741-750. doi:10.1016/j.tins.2015.09.003

58. Thompson JM, Neugebauer V. Amygdala Plasticity and Pain. Pain Res Manag. 2017;2017:1-12. doi:10.1155/2017/8296501

59. Janak PH, Tye KM. From circuits to behaviour in the amygdala. Nature. 2015;517(7534):284-292. doi:10.1038/nature14188

60. Kim MJ, Loucks RA, Palmer AL, et al. The structural and functional connectivity of the amygdala: from normal emotion to pathological anxiety. Behav Brain Res. 2011;223(2):403-410. doi:10.1016/j. bbr.2011.04.025

61. Michely J, Rigoli F, Rutledge RB, Hauser TU, Dolan RJ. Distinct processing of aversive experience in amygdala subregions. Biol Psychiatry Cogn Neurosci Neuroimaging. 2020;5(3):291-300. doi:10.1016/j.bpsc.2019.07.008

62. Corder G, Ahanonu B, Grewe BF, Wang D, Schnitzer MJ, Scherrer G. An amygdalar neural ensemble that encodes the unpleasantness of pain. Science. 2019;363(6424):276-281. doi:10.1126/ science.aap 8586

63. Ong AD, Thoemmes F, Ratner K, Ghezzi-Kopel K, Reid MC. Positive affect and chronic pain. Pain. 2020;161(6):1140-1149. doi:10.1097/j.pain.0000000000001828

64. Kim MJ, Mattek AM, Shin J. Amygdalostriatal coupling underpins positive but not negative coloring of ambiguous affect. Cogn Affect Behav Neurosci. 2020;17:1-12.

65. Letzen JE, Seminowicz DA, Campbell CM, Finan PH. Exploring the potential role of mesocorticolimbic circuitry in motivation for and adherence to chronic pain self-management interventions. Neurosci Biobehav Rev. 2019;98:10-17. doi:10.1016/j. neubiorev.2018.12.011 
66. Harris P, Loveman E, Clegg A, Easton S, Berry N. Systematic review of cognitive behavioural therapy for the management of headaches and migraines in adults. Br J Pain. 2015;9(4):213-224. doi:10.1177/ 2049463715578291

67. Seminowicz DA, Burrowes SAB, Kearson A, et al. Enhanced mindfulness-based stress reduction in episodic migraine. Pain. 2020;161(8):1837-1846. doi:10.1097/j.pain.0000000000001860
68. Bender R, Lange S. Adjusting for multiple testing - When and how? J Clin Epidemiol. 2001;54(4):343-349. doi:10.1016/S0895-4356(00) 00314-0

\section{Publish your work in this journal}

The Journal of Pain Research is an international, peer reviewed, open access, online journal that welcomes laboratory and clinical findings in the fields of pain research and the prevention and management of pain. Original research, reviews, symposium reports, hypothesis formation and commentaries are all considered for publication. The manuscript

Submit your manuscript here: https://www.dovepress.com/journal-of-pain-research-journa management system is completely online and includes a very quick and fair peer-review system, which is all easy to use. Visit http:// www.dovepress.com/testimonials.php to read real quotes from published authors. 